\title{
Electroreduction of Copper Dichloride Powder to Copper Nanoparticles in an Ionic Liquid
}

\author{
Tian Wu, ${ }^{1}$ Qing Huang, ${ }^{1}$ Wei Li, ${ }^{2}$ Gongxuan Chen, ${ }^{1}$ Xiaoling Ma, ${ }^{1}$ and Guoping Zeng ${ }^{1}$ \\ ${ }^{1}$ College of Chemistry and Life Science, Hubei University of Education, Wuhan 430205, China \\ ${ }^{2}$ Department of Rare Metals, Guangzhou Research Institute of Non-ferrous Metals, Guangzhou 510650, China \\ Correspondence should be addressed to Tian Wu; twu@whu.edu.cn and Xiaoling Ma; 240200025@qq.com
}

Received 11 November 2013; Accepted 20 December 2013; Published 6 February 2014

Academic Editor: Hui Xia

Copyright (c) 2014 Tian Wu et al. This is an open access article distributed under the Creative Commons Attribution License, which permits unrestricted use, distribution, and reproduction in any medium, provided the original work is properly cited.

\begin{abstract}
There were a large number of ionic liquids electrodeposition reported in the literature; but were still in the laboratory stage some problems in the practical application of electrodeposition remain such as easily reacted with moisture in the air $\left(\mathrm{AlCl}_{3}\right.$ ionic liquid), high cost, and corrosive (dialkylimidazolium cation and $\mathrm{BF}_{4}{ }^{-}, \mathrm{PF}_{6}{ }^{-}$ionic liquid). In addition to the above shortcomings, low solubility of many metal salts in ionic liquids limits the practical application. In order to solve the problem of low solubility, [Bmim $] \mathrm{Cl}$ could be added $[\mathrm{Bmim}] \mathrm{PF}_{6}$, which could significantly increase the solubility of metal chlorides; this method could be commonly used in preparing metal electrochemical reduction of metal chlorides. Our study showed that adding cationic groups in hydroxyl ionic liquid could cause the good solubility of transition metal chlorides, such as $\mathrm{CuCl}_{2}$. Complexation of hydroxyl functional group and transition metal ions increased solubility, resulting in a larger deposition current density and surface electrochemical reduction of copper nanoparticles deposited on the metal $\mathrm{Ni}$. The electroreduction mechanism and behavior of $\mathrm{CuCl}_{2}$ in hydroxyl ionic liquid and the $\mathrm{Cu}$ nanoparticle formation mechanism were investigated based on a comparison between similar experiments in the ionic liquid.
\end{abstract}

\section{Introduction}

Electrolytic metal electrodeposition method refers to a solid surface in the process to obtain the deposited metal layer. It aims for the following areas: the refining of the metal material, the surface characteristics changing of the solid material, and the preparation of a specific component of metal or semiconductor materials. Generally, metal electrodeposition includes electrolytic smelting, electroforming, and plating. As an electrochemical deposition medium, the application of aqueous solution was restricted by electrochemical window of water, while conventional molten salt is generally limited by high temperature and energy consumption. Compared with others, ionic liquids have thermal stability, nonvolatile, nonflammable, moderate conductivity, and wide electrochemical window nature and could compensate for the lack of water and molten salts $[1,2]$. Moreover, most of the metal deposited in the aqueous solution can be generated in ionic liquids, and some light metals, refractory metals, and semiconductor materials can only be obtained in ionic liquids at room temperature which could not get in aqueous deposition. Furthermore, the addition of many toxic agents in the conventional electroplating could generally be considered as a high environmental pollution industry. In ionic liquids for electroplating, it is no need to add in substances for suppression of hydrogen evolution and even toxic additives. Therefore, there is a hope for ionic liquids to become a kind of important green electrolyte in electrochemical preparation of metal, semiconductor materials, and electroplating [3].

Murase et al. [4] studied the oxidation-reduction of electrodeposition of copper in TMHA-Tf2N ionic liquids; this process involves the reaction of $\mathrm{Cu}+\mathrm{Cu}^{2+} \rightarrow 2 \mathrm{Cu}^{+}$. Finally, to obtain electrodeposition of copper on the cathode, the deposition process current efficiency of single-electron reaction reached almost $100 \%$. Chen and Sun [5] reported that copper was electrodeposited on polycrystalline tungsten electrode, platinum electrode, and a glassy carbon electrode in tetrafluoroborate 1-ethyl-3-methyl imidazole (EMIC-BF ${ }_{4}$ ) ionic liquids, which joined the cuprous chloride increase the solubility of $\mathrm{CuCl} ; \mathrm{Cu}^{+}$could be oxidized to $\mathrm{Cu}^{2+}$ or 
reduced to metallic copper; $\mathrm{Cu} / \mathrm{Cu}^{+}$redox demonstrated a quasireversible charge transfer process.

Endres and Schweizer [6] studied the electrodeposition of copper on $\mathrm{Au}$ (111) as the matrix at different temperatures in trifluoromethanesulfonate imide 1-butyl-1-methylpyridine $\left(\mathrm{BMPy}-\mathrm{Tf}_{2} \mathrm{~N}\right)$ ionic liquid and sediment layers was observed using a scanning tunneling electron microscopy. Experimental results showed that the majority of the copper compound in ionic liquids has low solubility, the anodic dissolution could increase copper ions in the solution. Study found that the curve showed a consistent feature and, with the increase of temperature and current of copper, the deposition potential becomes more positive.

Although there a large number of electrodeposition behavior of ionic liquids was reported in the literature [711], while most of them were still in the laboratory stage, the practical application of electrodeposition still has some problems, such as $\mathrm{AlCl}_{3}$ ionic liquid which can easily react with moisture in the air and should be operated in the glove box; ionic liquid is constituted of dialkyl imidazolium cation and $\mathrm{BF}_{4}{ }^{-}, \mathrm{PF}_{6}{ }^{-}$; although the stability is improved, the cost was high and could be corrosive when placed it for a long time. In addition, the low solubility of the metal salts in ionic liquids also limits the practical application.

Following the successful electrochemical reduction of solid metal oxides to the metals in high temperature molten salts [12-14], solid $\mathrm{CuO}$ nanoparticles were electroreduced to copper nanoparticles in an aqueous electrolyte, aiming for catalytic applications [14].

In order to solve the problem of low solubility, [Bmim] $\mathrm{Cl}$ can be added to $[\mathrm{Bmim}] \mathrm{PF}_{6}$, which can significantly increase the solubility of metal chlorides; the method could be commonly used in electrochemical reduction of metal from metal chlorides.

Ionic liquids usually contain functional groups and could be divided into functional ionic liquids and conventional ionic liquids. Function ionic liquid (task-specific ionic liquid, abbreviated as TSIL) has a specific group introduced into the structure, which was provided with a unique function. For example, cuprous chloride and cupric chloride were recently reported having a good solubility in 1-ethyl-3methylimidazolium dicyanamide ionic liquid, which may be due to the complexation of dicyandiamide anion. At the same time, electrochemical behavior of nickel chloride was also studied in such ionic liquid [15]. The emergence of functionalized ionic liquids enriches the types of ionic liquids and leads the direction of the development of ionic liquids [16]. Functionalized ionic liquids (FILs) containing alcohol on the alkyl arms have received much attention in the fields of organic synthesis and catalysis. Compared to traditional ILs, they show additional advantages such as alterable polarity, lower viscosity, and higher solubility for inorganic salts $[17,18]$. Since the $\mathrm{OH}$ group has the positive effect on the solubility, this idea can extend to the corresponding hydroxyl-functionalized ionic liquids (HFILs) directly for the increasing solubility of $\mathrm{CuCl}_{2}$ without using other additives. The solubility of transition metal halides can be increased by using cations that are capable of forming complex ions with transition metals. In view of the good donor ligand property that is known for the hydroxyl group, transition metal compounds should be soluble in the hydroxyl-functionalized ionic liquids (HFILs), and the preparation of a bath solution for electrodeposition is possible.

Although HFILs may be potentially useful electrolytes, examples using these RTILs for metal electrodeposition are very limited. In order to evaluate the utility of this new RTIL system, the electrochemistry and electrodeposition of copper in the HFILs were studied in this work. The voltammetric and nucleation behavior of copper electrodeposition at $\mathrm{Pt}$ and $\mathrm{Ni}$ electrodes, respectively, were studied (see Figure 5). Hydroxyl functional group and transition metal ions complexation increased the solubility, resulting in a larger deposition current density and surface electrochemical reduction of nanocopper particles deposited on metal Ni. And redox behavior of copper ions of $\mathrm{CuCl}_{2}$ in hydroxyl ionic liquid was different from the reported two single-electron redox behavior; three oxidation peaks was found in our experiment and the cause of the third oxidation peak were also analyzed. Electrodeposits of copper were prepared using controlled potential electrolysis at copper substrates and examined by scanning electron microscopy (SEM).

\section{Experimental}

2.1. Instruments and Reagents. The preparation and characterisation of 1-butyl-3-metylimidizolium chloride ([BMIM] $\mathrm{Cl}$, viscous liquid) and hexafluorophosphate ([BMIM] $\mathrm{PF}_{6}$, liquid) followed the literature description $[19,20]$. The powders of cuprous chloride $\left(\mathrm{CuCl}\right.$ : >97\%) and $\mathrm{CuCl}_{2}$ (99\%, Acros Organics) were thermally dried in vacuum before use. The solubility of these dried salts in [BMIM] $\mathrm{PF}_{6}$ was measured as follows. The IL was first saturated with an excess amount of the salt at $80^{\circ} \mathrm{C}$ under stirring for $48 \mathrm{~h}$. After standing still for over $24 \mathrm{~h}$, the clear solution was sampled at different temperatures $\left(80^{\circ} \mathrm{C}, 60^{\circ} \mathrm{C}, 40^{\circ} \mathrm{C}\right.$, and $\left.28^{\circ} \mathrm{C}\right)$. The sample was treated with concentrated $\mathrm{H}_{2} \mathrm{SO}_{4}$ and $\mathrm{H}_{2} \mathrm{O}_{2}$ and then diluted in double-distilled water before analysis by atomic absorption spectrometer (AAnalyst800, Perkin Elmer).

Electrochemical workstation: Shanghai $\mathrm{CH}$ Instrument Company, Chi 660A; Superheated tank: Shanghai Experimental Instrument Factory, ZKF030; Working electrode: $100 \mu \mathrm{m}$ Pt plate, $0.7 \mathrm{~mm} \mathrm{Cu}$ plate, $2 \mathrm{~mm} \times 5 \mathrm{mmNi}$ tablets; auxiliary electrode: $6 \mathrm{~mm} \times 4 \mathrm{~mm}$ Pt films, $6 \mathrm{~mm} \times 3 \mathrm{mmCu}$ tablets; reference electrode: $\mathrm{Ag} / \mathrm{Ag}^{+}$electrode; Electrolyzer: laminated glass structure, adding ionic liquid volume of approximately $1 \mathrm{~mL}$, with airway and seals.

The electrolytic products from the IL were washed with acetonitrile twice, dried in vacuum, and analysed directly by X-ray diffraction spectroscopy (XRD, SHIMADZU X-ray 6000 with $\mathrm{Cu}-\mathrm{K} \alpha$ ) and scanning electron microscopy (SEM, HITACHIx-650).

2.2. Making Reference Electrode. $\mathrm{AgPF}_{6}$ was dissolved in $\mathrm{BMIMPF}_{6}$, allowing the theoretical content of $\mathrm{Ag}^{+}$to be 3.0 Mm; a silver lining will be inserted into the solution and separated with the outer layer ionic liquid BMIMPF6 salt 


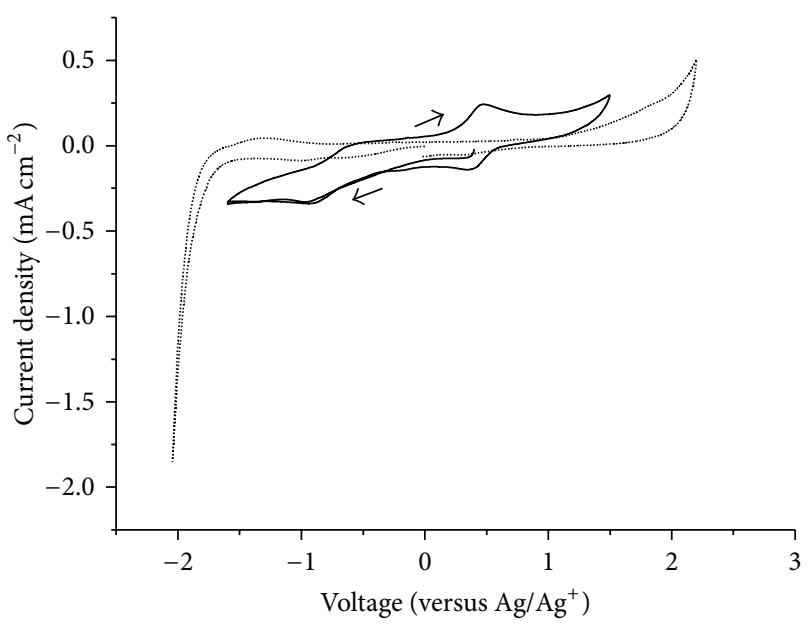

Figure 1: Cyclic voltammograms of a Pt-disk electrode (diameter: $100 \mu \mathrm{m}$ ) recorded under ambient conditions after addition of excess $\mathrm{CuCl}_{2}$ powder in the $\mathrm{BMIMPF}_{6}$ and standing for $1 \mathrm{~h}$.

bridge with alumina ceramic, then inserting secondary jacket into ion liquid.

2.3. Electrochemical Test Program. Ionic liquids were dried at $70^{\circ} \mathrm{C}, 12 \mathrm{~h}$ in vacuum prior to the electrochemical test; anhydrous cuprous chloride and cupric chloride were used without further processing. The copper ionic liquid was added and stirred under argon, through circulating the water temperature to the required test temperature. All the electrochemical tests should maintain the liquid level in the argon atmosphere.

Cyclic voltammetry tests were performed in a threeelectrode system; $\mathrm{Ag} / \mathrm{Ag}^{+}$was selected as the reference electrode; a large area platinum sheet as counter electrode, a platinum plate, copper plate and nickel plate as working electrode respectively

2.4. Constant Potential Electrolysis. A large area of copper was taken as the counter electrode, the working electrode was a nickel plate or copper plate. Powder on the nickel sheet samples were deposited with distilled water and acetone, then dried and prepared as samples for SEM morphology image analysis.

\section{Results and Discussion}

3.1. Electrochemical Behavior of $\mathrm{CuCl}_{2}$ in Ionic Liquids $\mathrm{BMIMPF}_{6}$. Electrochemical window of BMIMPF6 in an argon gas atmosphere was about $4.1 \mathrm{~V}$; no impurity peaks were found. After adding $\mathrm{CuCl}_{2}$, the mixture was stirred with $\mathrm{BMIMPF}_{6}$ at $40^{\circ} \mathrm{C}, 1 \mathrm{~h}$, and divalent copper ions were detected with electrochemical method in the system. As shown in Figure 1, the current peaks can be attributed to electrochemical conversion between these $\mathrm{Cu}, \mathrm{Cu}^{+}$, and $\mathrm{Cu}^{2+}$ ions. The quasireversible redox couple at about $0.5 \mathrm{~V}$ (versus $\mathrm{Ag} / \mathrm{Ag}^{+}$) was represented to the reaction $\mathrm{Cu}^{2+}+\mathrm{e}$ $\rightleftarrows \mathrm{Cu}^{+}$. The oxidation peak of $-0.6 \mathrm{~V}$, below potential of $-0.8 \mathrm{~V}$, corresponds to metallic copper reoxidation on the

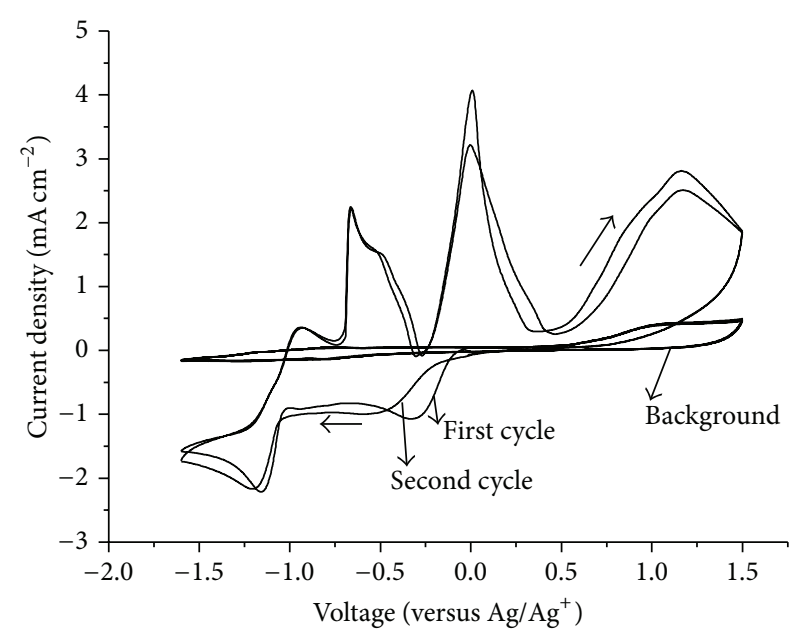

FIgure 2: Cyclic voltammograms of a Pt-disk electrode (diameter: $100 \mu \mathrm{m}$ ) recorded under ambient conditions after addition of excess $\mathrm{CuCl}_{2}$ powder in the $\mathrm{C}_{3} \mathrm{OHmimBF}_{4}$ and standing for $1 \mathrm{~h}$ at $60^{\circ} \mathrm{C}$. Potential scan rate: $50 \mathrm{mVs}^{-1}$.

platinum electrode. It is noted that current density at $1.5 \mathrm{~V}$ was increasing due to the oxidation of dissolved chloride ion in the system.

Compared with phenomena reported by $\mathrm{Yu}$ et al. that, after stirring $\mathrm{CuCl}$ overnight with $\mathrm{BMIMPF}_{6}$, no $\mathrm{Cu}^{+}$or $\mathrm{Cl}^{-}$ peaks were found in cyclic voltammetry, we speculate that the solubility of $\mathrm{CuCl}_{2}$ in BMIMPF6 was larger than $\mathrm{CuCl}$, comply, with inference of solubility size measured by atomic absorption spectrometry.

3.2. Cyclic Voltammetric Behavior of $\mathrm{C}_{3} \mathrm{OHmimBF}_{4} / \mathrm{CuCl}_{2}$ System. After slight excess of $\mathrm{CuCl}_{2}$ was added to $\mathrm{C}_{3} \mathrm{OHmimBF}_{4}$, copper ion concentration could be measured by a weighing method to be about $40 \mathrm{Mm}$. After strong stirring for 1 hour and standing after a short time, cyclic voltammetry study of the system was shown in Figure 2. The behavior cyclic voltammetry was significantly different from our previous observations of $\mathrm{CuCl}_{2}$ in BMIMPF6. Oxidation of chloride ion in $\mathrm{C}_{3} \mathrm{OHmimBF}_{4}$ was carried out at a lower electric potential of the oxidation reaction occurrence; the oxidation peak significantly decreases to $1.0 \mathrm{~V}$. For cupric ions and significantly different from the previously observed redox groups, two reduction peaks and three oxidation peaks were observed, respectively. The cause for the third oxidation peak was the focus of our research. Furthermore, the peak current density of both the chloride ions, and copper ions were significantly increased, which was closely related to greater solubility of $\mathrm{CuCl}_{2}$ in $\mathrm{C}_{3} \mathrm{OHmimBF}_{4}$.

As the scan range of Figure 2 includes the oxidation of chloride ions, in order to avoid the influence of chloride ions to copper oxide, interval between $-1.6 \mathrm{~V}$ and $0.4 \mathrm{~V}$ was chosen for a separate scan as shown in Figure 3. The system's open-circuit potential was at $0.1 \mathrm{~V}$, the reactions before the reduction peaks occurred as follows: in $0.1 \mathrm{~V} \sim-0.5 \mathrm{~V}, \mathrm{Cu}^{2+} \rightarrow \mathrm{Cu}^{+}$; in $-1 \mathrm{~V} \sim-1.6 \mathrm{~V}, \mathrm{Cu}^{+} \rightarrow \mathrm{Cu}$ (before the start of the reduction reaction, the concentration of chloride ion electrode surface was limited, and there was 


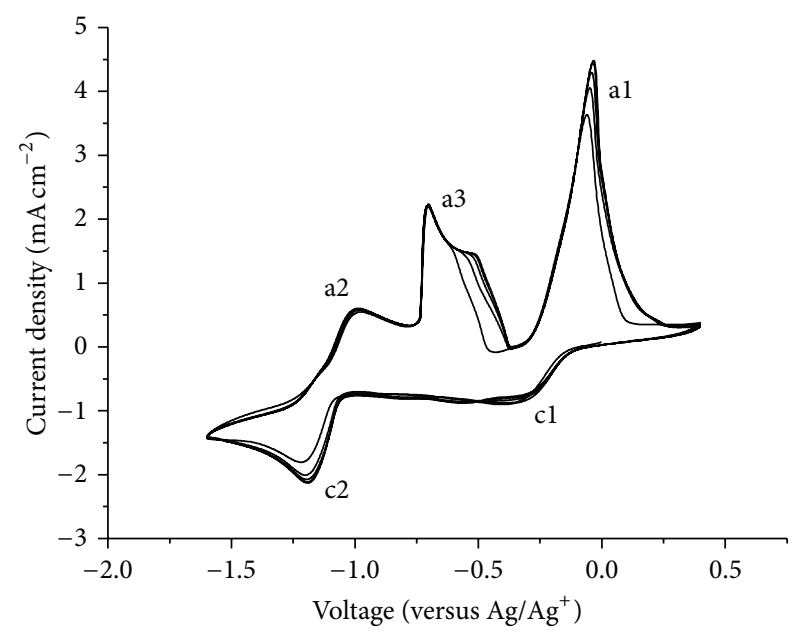

Figure 3: Cyclic voltammograms of a Pt-disk electrode (diameter: $100 \mu \mathrm{m}$ ) recorded under ambient conditions after addition of excess $\mathrm{CuCl}_{2}$ powder in the $\mathrm{C}_{3} \mathrm{OHmimBF}_{4}$ and standing for $1 \mathrm{~h}$ at $60^{\circ} \mathrm{C}$. Potential scan rate: $50 \mathrm{mVs}^{-1}$. Scanning range: $-1.6 \mathrm{~V} \sim 0.4 \mathrm{~V}$.

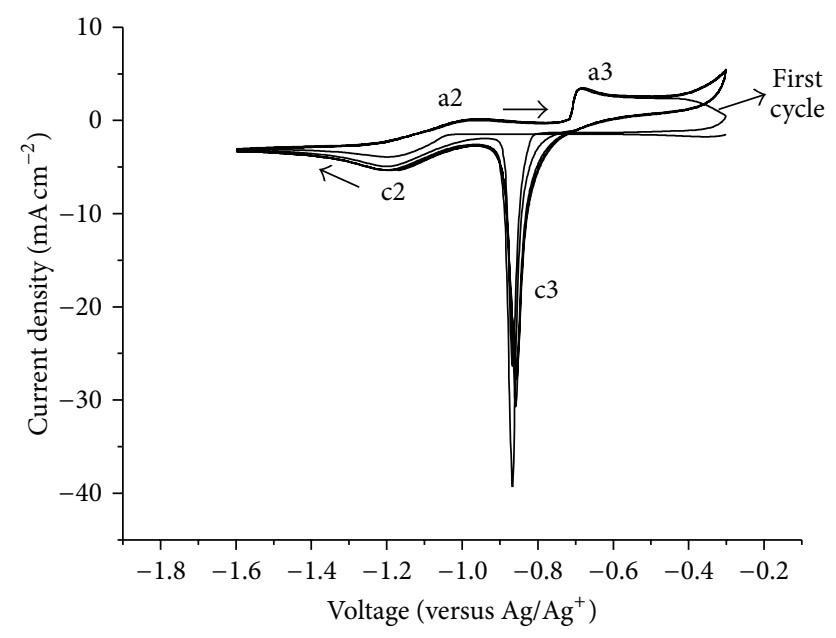

FIGURe 4: Cyclic voltammograms of a Pt-disk electrode (diameter: $100 \mu \mathrm{m}$ ) recorded under ambient conditions after addition of excess $\mathrm{CuCl}_{2}$ powder in the $\mathrm{C}_{3} \mathrm{OHmimBF}_{4}$ and standing for $1 \mathrm{~h}$ at $60^{\circ} \mathrm{C}$. Potential scan rate: $50 \mathrm{mVs}^{-1}$. Scanning range: $-1.6 \mathrm{~V} \sim-0.3 \mathrm{~V}$.

a lot of $\mathrm{C}_{3} \mathrm{OHmim}^{+}$in the system, so here changes of the valence of copper ions are complexed of $\mathrm{C}_{3} \mathrm{OHmin}+$ to form hydroxy complexes). In order to determine the attribution of the respective redox peaks, the interval between $-1.6 \sim-0.3 \mathrm{~V}$ was shown in Figure 4. Figure 6 further confirmed the $\mathrm{Cu}+2$ to +1 transition between prices.

In Figure 4, we have retraced the oxidation potential to $-0.3 \mathrm{~V}$, which was not readily oxidized to the peak position al, resulting in a significant change in the second week of a sharp peak $\mathrm{c} 3$, which could be interpreted as $\mathrm{Cu}^{2+}$ scanning in the first week after the reduction of $\mathrm{Cu}$ and generated free chloride ion near the electrode, which undergone a copper competition with a hydroxyl ligand system. The product of the oxidation peak a3 was complexed with the hydroxyl and $\mathrm{Cu}^{+}$. Soon, afterwards, further complexation for $[\mathrm{Cu}$

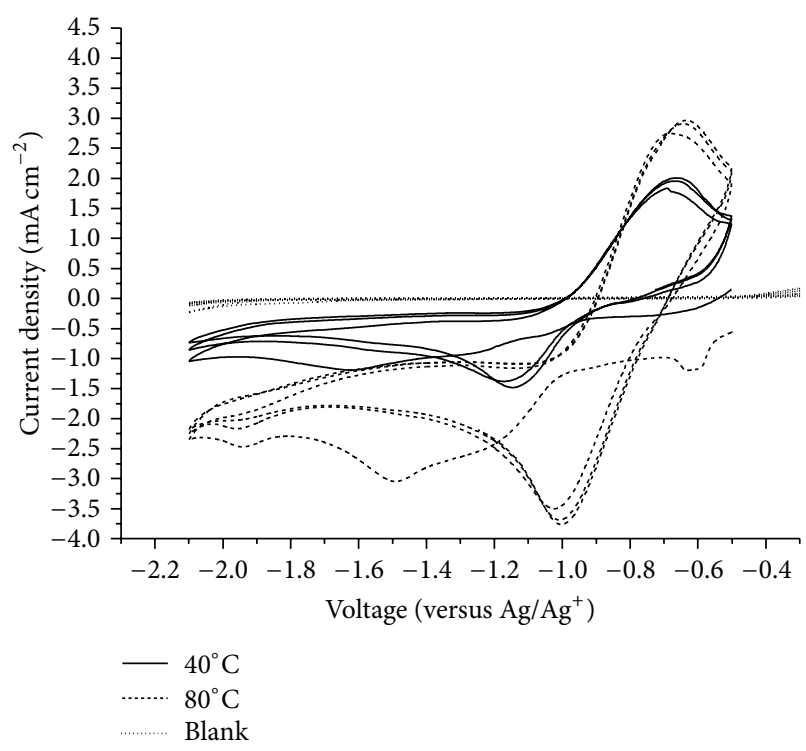

FIGURE 5: Cyclic voltammograms of a Ni electrode recorded under ambient conditions after addition of excess $\mathrm{CuCl}_{2}$ powder in the $\mathrm{C}_{3} \mathrm{OHmimBF}_{4} / \mathrm{Cl}$ and standing at $60^{\circ} \mathrm{C}$ and $80^{\circ} \mathrm{C}$. Potential scan rate: $50 \mathrm{mVs}^{-1}$. Scanning range: $-1.6 \mathrm{~V} \sim 0.4 \mathrm{~V}$.

$\left.\left(\mathrm{C}_{3} \mathrm{OHmim}-\mathrm{BF}_{4}^{-}\right)\right]$and chloride ion around the electrode occurred. And a 3 and $\mathrm{c} 3$ constituted a group of chloride ion in the dissolution peak ligand as follows: $\left[\mathrm{Cu}\left(\mathrm{C}_{3} \mathrm{OHmim}-\right.\right.$ $\left.\left.\mathrm{BF}_{4}{ }^{-}\right)\right]+\mathrm{Cl}^{-}(\mathrm{l})-\mathrm{e} \rightarrow\left[\mathrm{Cu}\left(\mathrm{C}_{3} \mathrm{OHmim}-\mathrm{BF}_{4}{ }^{-}\right) \mathrm{Cl}\right]^{+}$. Noting the changes in the redox battery, during the first week peak areas a3 was larger following in the second week of a sharp peak c3 and the corresponding peak area a3 was significantly reduced.

\subsection{Electrodeposition of Copper Chloride on Nickel Sheet}

3.3.1. Electrochemical Behavior of $\mathrm{CuCl}_{2}$ in the $\mathrm{C}_{3} \mathrm{OHMIMBF}_{4} / \mathrm{Cl}$. Reduction peak of the first cycle which is more complex and may be present on the surface of nickel electrode reduced oxidizing substances. Subsequent cycles showed a set of simple redox peaks of copper ions in the reduction behavior of nickel electrode on a platinum electrode with a significant difference. Only a bunch of redox peaks were observed, represent cupric two-electron redox process. If rising the temperature, copper-nickel electrode reduction peak current has been significant negative shifted, the oxidation peak position basically unchanged. It could avoid oxidation which leads to excessive current positive nickel electrode oxidation. We examined the reduction potential, temperature, and reduction time on the product morphology.

3.3.2. SEM Figures of Products of Constant Potential Electrolysis in $\mathrm{CuCl}_{2} / \mathrm{C}_{3} \mathrm{OHMIMBF} / \mathrm{Cl}$ System. SEM images of the copper nanoparticles prepared by electro-reduction of the $\mathrm{CuCl}_{2}$ powder on a $\mathrm{Ni}$ foil in $\mathrm{C}_{3} \mathrm{OHmimBF}_{4} / \mathrm{Cl}$ at (a) $-1.7 \mathrm{~V}$, $80^{\circ} \mathrm{C}, 400 \mathrm{~s}$; (b) $-1.5 \mathrm{~V}, 80^{\circ} \mathrm{C}, 400 \mathrm{~s}$; (c) $-1.7 \mathrm{~V}, 40^{\circ} \mathrm{C}, 1200 \mathrm{~s}$; and (d) $-1.8 \mathrm{~V}, 40^{\circ} \mathrm{C}, 1200 \mathrm{~s}$ (versus $\mathrm{Ag} / \mathrm{Ag}^{+}$) were shown in Figure 6. At $80^{\circ} \mathrm{C}$, particles were approximately $50 \mathrm{~nm}$ and piled in large particle sizes ranging from about several 


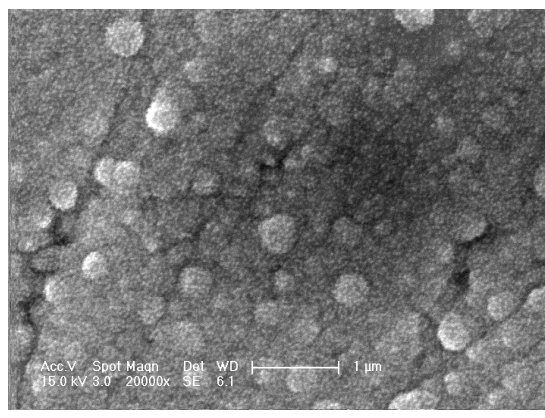

(a)

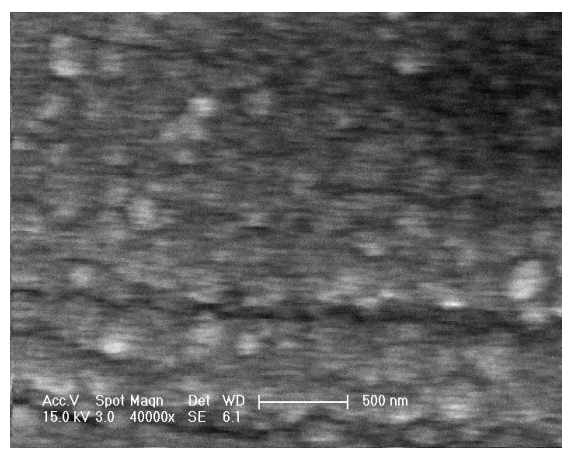

(c)

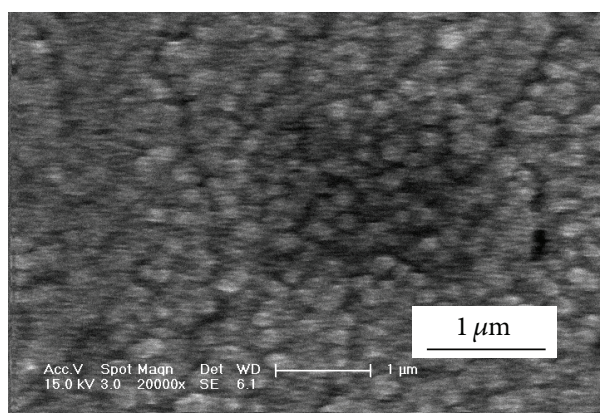

(b)

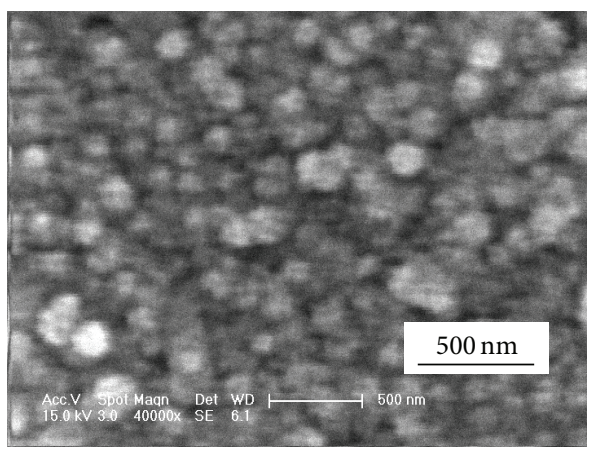

(d)

FiguRE 6: SEM images of the copper nanoparticles prepared by electro-reduction of the $\mathrm{CuCl}_{2}$ powder on a $\mathrm{Ni}$ foil in $\mathrm{C}_{3} \mathrm{OHmimBF}_{4} / \mathrm{Cl}_{\text {at }}$ (a) $-1.7 \mathrm{~V}, 80^{\circ} \mathrm{C}, 400 \mathrm{~s}$; (b) $-1.5 \mathrm{~V}, 80^{\circ} \mathrm{C}, 400 \mathrm{~s}$; (c) $-1.7 \mathrm{~V}, 40^{\circ} \mathrm{C}, 1200 \mathrm{~s}$; and (d) $-1.8 \mathrm{~V}, 40^{\circ} \mathrm{C}, 1200 \mathrm{~s}$ (versus $\mathrm{Ag} / \mathrm{Ag}^{+}$).

hundred nanometers. When the potential rose up to $-1.5 \mathrm{~V}$, particles were about $50 \mathrm{~nm}$ and piled in large particle size uniformity, about $300 \mathrm{~nm}$. When deposition potentials were more positive, more uniform particles could be obtained, the deposition layer was in smaller size. When deposition potential becomes negative, the particle size becomes larger. Figure 6(c) represented a figure large particle size decreases (the same deposition potential, at different temperatures). Figures 6(c) and 6(d) showed that particle size increased slightly compared with large particle size was relatively uniform, about $300 \mathrm{~nm}$. When the temperature of deposition was lower, smaller and more uniform deposited layer could be obtained.

\section{Summary}

Our study shows that cationic group to add a hydroxylfunctionalized ionic liquids for the transition metal chlorides, such as $\mathrm{CuCl}_{2}$, has good solubility. Complexation between hydroxyl functional group and transition metal ions increased solubility, resulting in a larger deposition current density. $\mathrm{CuCl}_{2}$ hydroxyl ions in the liquid redox behavior of copper ions with the reported two single-electron redox behavior were different; we got three oxidation peaks, and the cause analysis of the third oxidation peak was made.

The difference of nickel electrode $\mathrm{CuCl}_{2}$ reduction behavior in the platinum electrode was studied. Only one pair of redox peaks was observed on nickel electrode corresponding to divalent copper two-electron redox process. Nanocopper particles were electrochemically reduced and deposited on the surface of metal Ni. When deposition potential was more positive, more uniform particles were obtained and the deposition layer was in smaller size.

\section{Conflict of Interests}

The authors declare that there is no conflict of interests regarding the publication of this paper.

\section{Acknowledgments}

The authors acknowledge the financial support from the National Natural Science Foundation of China (Grant nos. 21303045 and 51204060) the Hubei University of Education Key Disciplines (Applied Chemistry), "Excellent Teacher Team Building" Scientific Research Project of Hubei University of Education (Grant no. 2012K103), Open Fund of Key Laboratory of Hubei Province of Implantation of Anticancer Active Substances Purification and Application and the Natural Science Foundation of Guangdong Province (no. S2012040007501).

\section{References}

[1] P. Wasserscheid and T. Welton, Ionic Liquids in Synthesis, WileyVCH Verlag GmbH \& Co. KGaA, 2002.

[2] T. Welton, "Room-temperature ionic liquids. Solvents for synthesis and catalysis," Chemical Reviews, vol. 99, no. 8, pp. 20712083, 1999. 
[3] F. Endres, "Ionic liquids: solvents for the electrodeposition of metals and semiconductors," ChemPhysChem, vol. 3, no. 2, pp. 144-154, 2002.

[4] K. Murase, K. Nitta, T. Hirato, and Y. Awakura, "Electrochemical behaviour of copper in trimethyl-n-hexylammonium bis ((trifluoromethyl)sulfonyl)amide, an ammonium imide-type room temperature molten salt," Journal of Applied Electrochemistry, vol. 31, no. 10, pp. 1089-1094, 2001.

[5] P.-Y. Chen and I.-W. Sun, "Electrochemical study of copper in a basic 1-ethyl-3-methylimidazolium tetrafluoroborate room temperature molten salt," Electrochimica Acta, vol. 45, no. 3, pp. 441-450, 1999.

[6] F. Endres and A. Schweizer, "The electrodeposition of copper on $\mathrm{Au}(111)$ and on HOPG from the $66 / 34 \mathrm{~mol} \%$ aluminium chloride/1-butyl-3-methylimidazolium chloride room temperature molten salt: an EC-STM study," Physical Chemistry Chemical Physics, vol. 2, no. 23, pp. 5455-5462, 2000.

[7] W. R. Pitner and C. L. Hussey, "Electrodeposition of zinc from the Lewis acidic aluminum chloride-1-methyl-3-ethylimidazolium chloride room temperature molten salt," Journal of the Electrochemical Society, vol. 144, no. 9, pp. 3095-3103, 1997.

[8] P.-Y. Chen and I.-W. Sun, "Electrodeposition of cobalt and zinccobalt alloys from a lewis acidic zinc chloride-l-ethyl-3-methylimidazolium chloride molten salt," Electrochimica Acta, vol. 46, no. 8, pp. 1169-1177, 2001.

[9] Y. Katayama, S. Dan, T. Miura, and T. Kishi, "Electrochemical behavior of silver in 1-ethyl-3-methylimidazolium tetrafluoroborate molten salt," Journal of the Electrochemical Society, vol. 148, no. 2, pp. C102-C105, 2001.

[10] Q. Liao, W. R. Pitner, G. Stewart, C. L. Hussey, and G. R. Stafford, "Electrodeposition of aluminum from the aluminum chloride-1-methyl-3-ethylimidazolium chloride room temperature molten salt + benzene," Journal of the Electrochemical Society, vol. 144, no. 3, pp. 936-943, 1997.

[11] G. E. Gray, P. A. Kohl, and J. Winnick, "Stability of sodium electrodeposited from a room temperature chloroaluminate molten salt," Journal of the Electrochemical Society, vol. 142, no. 11, pp. 3636-3642, 1995.

[12] G. Z. Chen, D. J. Fray, and T. W. Farthing, "Direct electrochemical reduction of titanium dioxide to titanium in molten calcium chloride," Nature, vol. 407, no. 6802, pp. 361-364, 2000.

[13] X. Jin, P. Gao, D. Wang, X. Hu, and G. Z. Chen, "Electrochemical preparation of silicon and its alloys from solid oxides in molten calcium chloride," Angewandte Chemie International Edition, vol. 43, no. 6, pp. 733-736, 2004.

[14] W.-K. Han, J.-W. Choi, G.-H. Hwang, S.-J. Hong, J.-S. Lee, and S.-G. Kang, "Fabrication of $\mathrm{Cu}$ nano particles by direct electrochemical reduction from $\mathrm{CuO}$ nano particles," Applied Surface Science, vol. 252, no. 8, pp. 2832-2838, 2006.

[15] M.-J. Deng, I.-W. Sun, P.-Y. Chen, J.-K. Chang, and W.-T. Tsai, "Electrodeposition behavior of nickel in the water- and airstable 1-ethyl-3-methylimidazolium-dicyanamide room-temperature ionic liquid," Electrochimica Acta, vol. 53, no. 19, pp. 5812-5818, 2008.

[16] A. Metlen and R. D. Rogers, “The second evolution of ionic liquids: from solvents and separations to advanced materialsenergetic examples from the ionic liquid cookbook," Accounts of Chemical Research, vol. 40, pp. 1182-1192, 2007.

[17] S. Zhang, X. Qi, X. Ma, L. Lu, and Y. Deng, "Hydroxyl ionic liquids: the differentiating effect of hydroxyl on polarity due to ionic hydrogen bonds between hydroxyl and anions," Journal of Physical Chemistry B, vol. 114, no. 11, pp. 3912-3920, 2010.
[18] X. Wei, L. Yu, X. Jin, D. Wang, and G. Z. Chen, "Solar-thermochromism of pseudocrystalline nanodroplets of ionic liquidNiII complexes immobilized inside translucent microporous PVDF films," Advanced Materials, vol. 21, no. 7, pp. 776-780, 2009.

[19] H. Sun, L. Yu, X. Jin, X. Hu, D. Wang, and G. Z. Chen, "Unusual anodic behaviour of chloride ion in 1-butyl-3-methylimidazolium hexafluorophosphate," Electrochemistry Communications, vol. 7, no. 7, pp. 685-691, 2005.

[20] J. G. Huddleston, A. E. Visser, W. M. Reichert, H. D. Willauer, G. A. Broker, and R. D. Rogers, "Characterization and comparison of hydrophilic and hydrophobic room temperature ionic liquids incorporating the imidazolium cation," Green Chemistry, vol. 3, no. 4, pp. 156-164, 2001. 

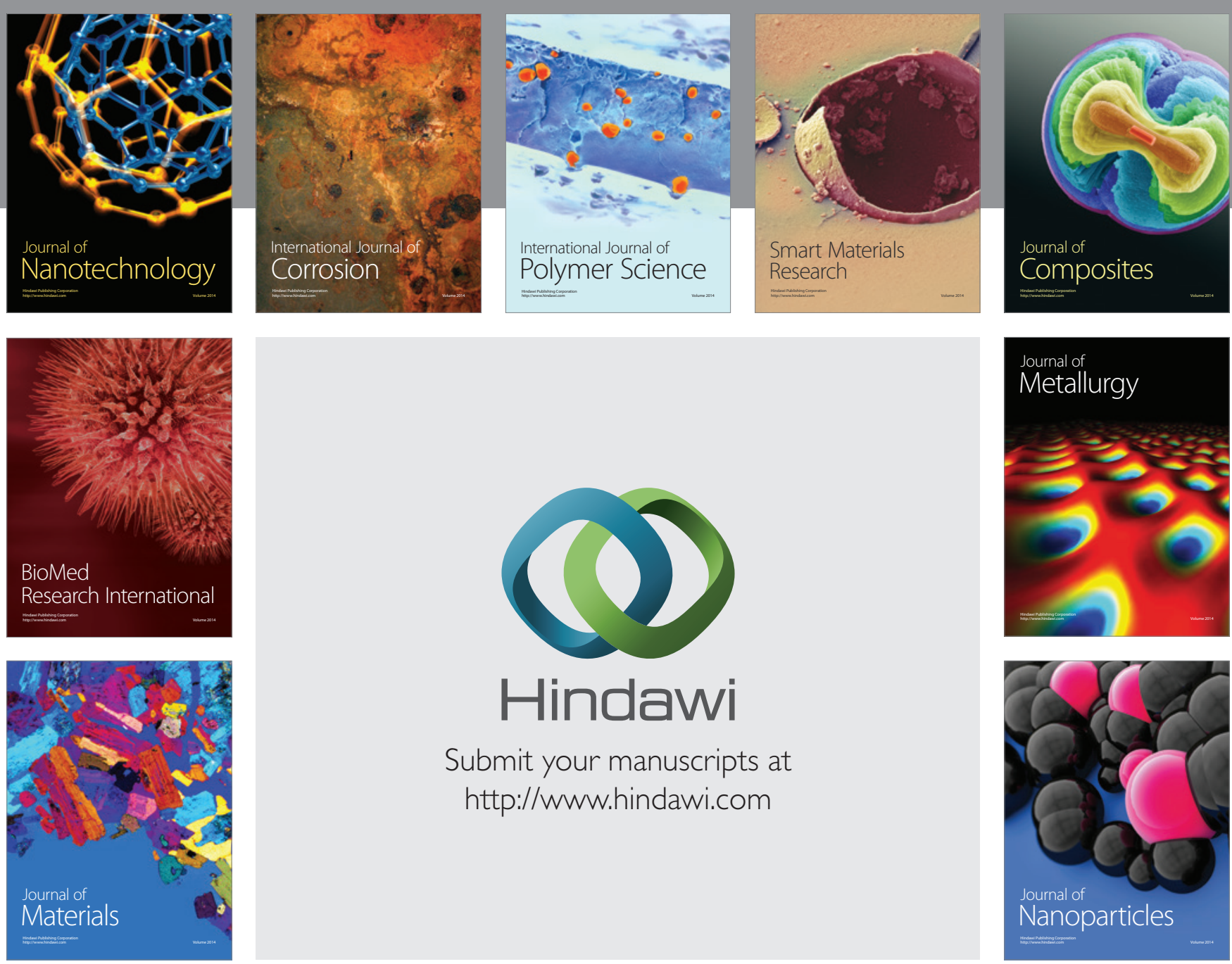

Submit your manuscripts at http://www.hindawi.com
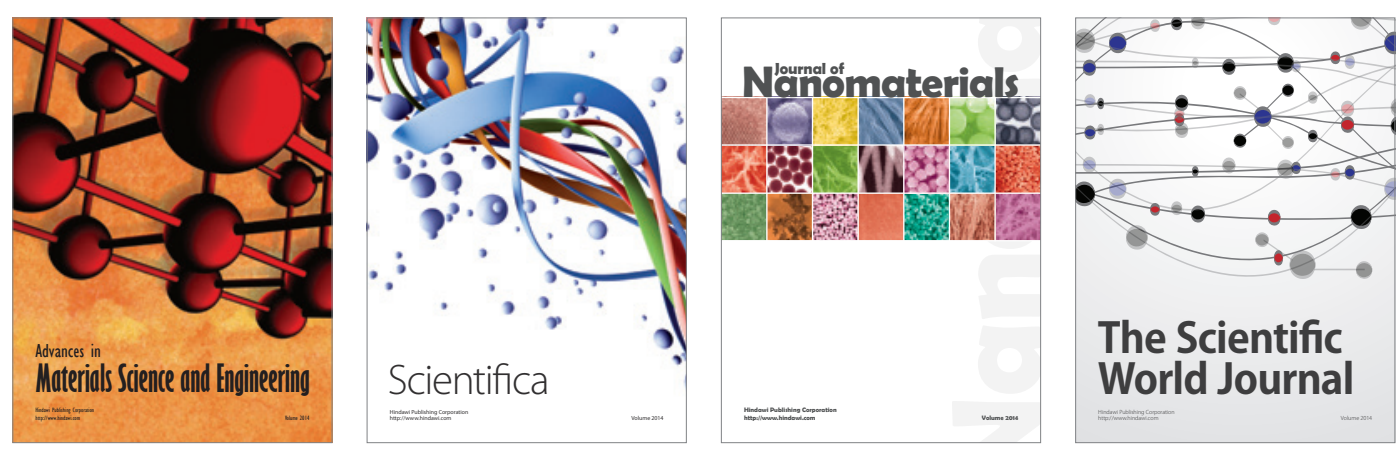

\section{The Scientific World Journal}
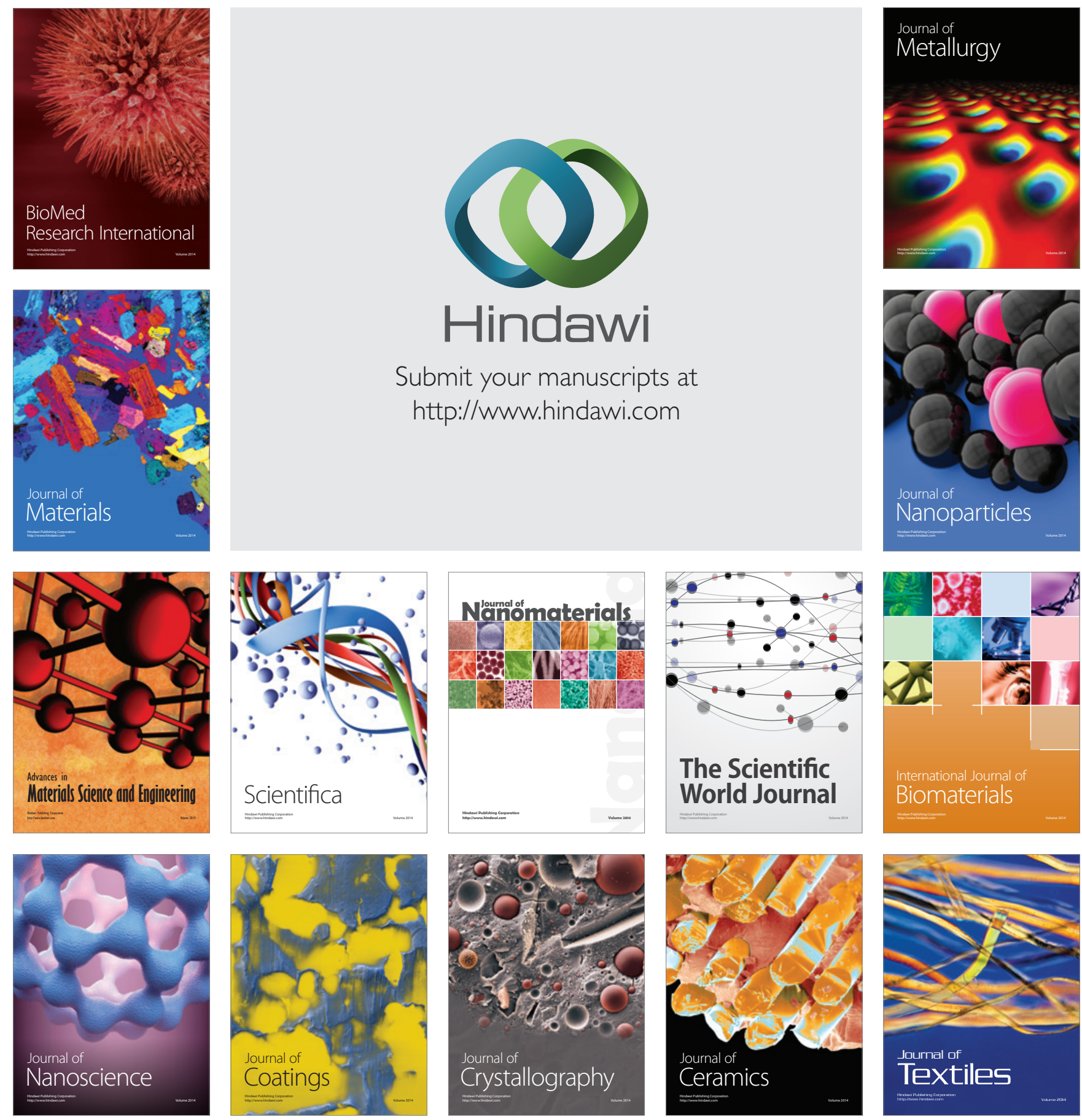